\title{
Development of Time Registration and New Criteria
}

\author{
Andris Buiks* and Alberts Aldersons \\ Department Institute of Mathematics and Computer Science, Latvian University, Latvia
}

Received: 制 July 10, 2018; Published: 制 July 12, 2018

*Corresponding author: Andris Buiks, Department Institute of Mathematics and Computer Science, Latvian University, Raingabulv, Latvia

\section{Abstract}

The paper is established for use in medicine, psychology, in man's self-development training, breathing technique's training, in the field of stress resistance, health promotion, strengthening of the capacity of work. We involve new technology for registration of time interval between two consecutive EKG RR intervals (R peaks):

a) The time between two consecutive R peaks, and

b) Time interval from the beginning of registration and beginning of each whole some R or pulsogram's peak. Our new mathematical algorithm allows reconstructing all pulsograms or RR intervalograms, providing full use of time domain and also frequency domain methods. Here we give a short review of our works.

Keywords: Heart Rate Variability; RR Interval; Time Interval; Cubic Spline; Empty Interval

\section{Introduction}

The paper is created for use in medicine, psychology, in man's self-development training, breathing technique's training, in the field of stress resistance, health promotion, strengthening of the capacity for work; and it relates to the apparatus and methods for detection of the heart rate variability and it may be used in providing biofeedback during training sessions of organism's vegetative balance and coherence [1-4]. The most prominent data analysis method - frequency oftime domain method needs very precise data acquisition technology, which is difficult to obtain, especially in practical everyday conditions, for example - in gym centres, individual biofeedback devices or apparatus for measurement of blood pressure and pulse. We involve new technology for registration of time interval between two consecutive EKG RR intervals or pulse wave peaks, which consist of simultaneous registration of two time intervals: 1) the time between RR intervals, and 2) time interval from the beginning of registration and beginning of each wholesome $\mathrm{R}$ orpulsogram'speak. Our new mathematical algorithm allows reconstructing approximate all pulsograms or RR intervalograms, providing full use of time domain methods.

Contrary to the view that under optimal conditions the heart beats sequence should remind the metronome, this is definitely not so [5]. Due to influence of Autonomic Nervous System, affecting the sinus node (nervous centre, located in the heart, which activates each of the next cardiac cycle starting after a pause), pulse beats followed each other at different time intervals, and, as a result, the time span between two consecutive heart beats can vary over a wide range - from 400 to 1500 msec. Plotting these following time intervals graphically, we get a wavy line. It is called the Heart Rate Variability (HRV) line. It turned out that this curve is very informative [6]. When registering a heartbeat, we get a simple series of numbers (intervals between every two consecutive heartbeats in millisecond's, in average 70 numbers during minute) from which we may derive many different indicators of the activity of vegetative nervous system, and, in addition, each of these numbers are characterized by strongly different physiological or psychological conditions. That is why we see a rapid increase of searches of new algorithms, approbation of new mathematical models. Any technique that allows you to record an electrocardiogram is valid. As recording equipment does not play important role for the conditions (as it for many other so called psycho physiological methods, for example, galvanic skin response), the technical details of the HRV record is no longer even object for serious discussions in the scientific literature.

We should start with the fact that the HRV was one of the chief methods used for evaluation of physiological state in aerospace medicine and psychology (it was in the period around years 19501980, mainly in Russia [6]). There are many studies that indicate the relationship between emotions and changed SRV indicators. HRV may be used as an indicator of risk prediction after myocardial infarction [6]. Until about 1980-ties the only methods of, primary used in direct time regime were time domain methods, where some un-precocity of data registration may be acceptable, somehow a great amount of very sophistically methods for registration process errors corrections were elaborated. Nevertheless, it was useful practice to simply delete the wrong fragments of registered signals. 
Doctor Alderson and I spent around twenty years working together. Our first work was to obtain the Latvian patent in year 2008. Then we decided to write an article [2] publishing the patent algorithm. It is a popular article of the lesions. Following Research Gate data, it has been read by around 300 researchers.

The situation changes when the frequency domain method for RR interval analysis enters. Due to sophistically mathematical algorithms used in frequency domain methods, also the demand for data acquisition quality remarkably grows up. But the article's weakness is that it compares only two adjacent heart beats. In the next article [3] we go deeper, and give further algorithm. And then we describe the process of time from the beginning of the process and see new criteria [4]. It has been read by around 100 researchers. We approximate the empty intervals by cubic spline approximation [5,7].

\section{Conclusion}

We have constructed some new form of registration of the time interval between the current and the previous heartbeats and the time from the beginning of the registration process. We have some new spline approximation by splines for the reconstruction of lost heartbeats. This allows displaying blank intervals in the drawing. The usage of spline ensures the smoothness of the obtained curve, because the cubic spline has a continuous second derivative.In the future we hope to make extrapolation to look into the upcoming

\section{ISSN: 2574-1241}

DOI: 10.26717/BJSTR.2018.06.001395

AndrisBuiks. Biomed J Sci \& Tech Res

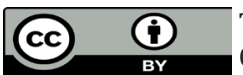

This work is licensed under Creative Commons Attribution 4.0 License

Submission Link: https://biomedres.us/submit-manuscript.php intervals. It is hard to see now how long this interval should be: it could be from five until unlimited beats.

\section{References}

1. Buikis A, Aldersons A (2008) Training method for promotion of emotional stress reduction, psychological coherence and vegetative balance. Latvian patent No. 13729, Riga p. 14.

2. Aldersons A, Buikis A (2011) Mathematical algorithm for heart rate variability analysis. Recent Advances in Applied \& Biomedical Informatics and Computational Engineering in Systems Applications pp. 381-386.

3. Buikis A, Aldersons A (2017) In depth mathematical algorithm for heart rate variability analysis. Journal of Multidisciplinary Engineering Science Studies 3(1): 1262-1269.

4. Buikis A, Aldersons A (2017) Time Registration and Life Science Data Registration. International Journal of Biology and Biomedicine 4(5): 201-208.

5. Buikis A, Buike M (2015) The Conservative Averaging Method: applications, theory and new hyperbolic approximation. Mathematical and Computational Methods in Applied Sciences. Proceedings of the $3^{\text {rd }}$ International Conference on Applied, Numerical and Computational Mathematics. Sliema, Malta, 17-19: 58-67.

6. (1996) Heart rate variability. Standards of measurement, psychological interpretation, and clinical use. Task force of the European Society of Cardiology the North American Society of Pacing Electrophysiology. Circulation 93(5): 1043-1065.

7. Parin VV, Baevskij PM, Volkov YN, Gazenko OG (1967) Cosmic cardiology. Russia.

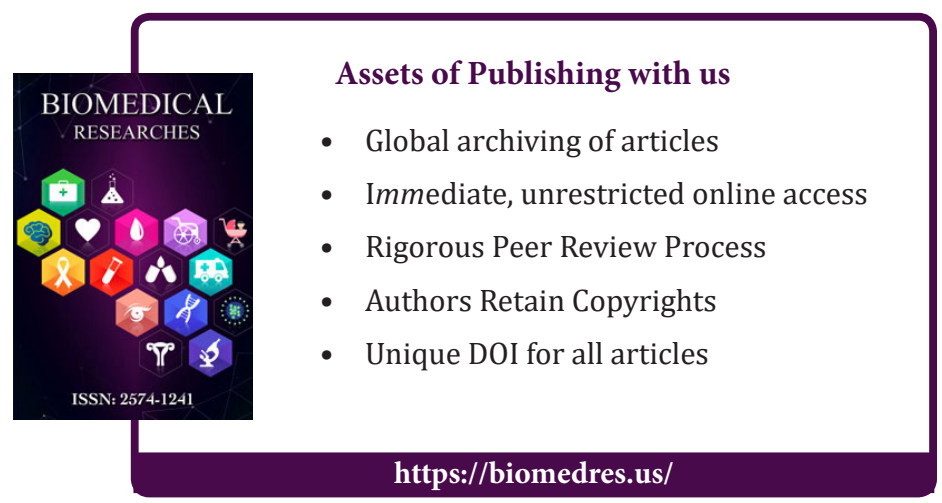

Cite this article: Andris B, Alberts A. Development of Time Registration and NewCriteria Biomed J Sci\&Tech Res 6(4)- 2018. BJSTR. 